\title{
Myelin Oligodendrocyte Glycoprotein (MOG) Antibody Disease in a 11-year-old child with COVID 19 infection
}

Archana Khan ( $\sim$ arch.agr@gmail.com )

NH SRCC Children's Hospital, Mumbai, India https://orcid.org/0000-0003-0842-2198

Hiren Panwala

NH SRCC Children's Hospital, Mumbai, India https://orcid.org/0000-0003-3718-8708

Divya Ramadoss

NH SRCC Children's Hospital, Mumbai, India https://orcid.org/0000-0002-0782-6142

Raju Khubchandani

NH SRCC Children's Hospital, Mumbai, India https://orcid.org/0000-0003-4543-901X

\section{Case Report}

Keywords: COVID 19, Myelin Oligodendrocyte Glycoprotein Antibody Disease, Optic Neuritis

Posted Date: November 10th, 2020

DOI: https://doi.org/10.21203/rs.3.rs-104308/v1

License: (a) (i) This work is licensed under a Creative Commons Attribution 4.0 International License. Read Full License

Version of Record: A version of this preprint was published at Indian Journal of Pediatrics on January 20th, 2021. See the published version at https://doi.org/10.1007/s12098-020-03656-7. 


\section{Abstract}

Optic Neuritis is the most common presentation of MOG Antibody Disease (MOG-AD). We share our experience with a 11-year-old boy who developed MOG associated Optic Neuritis temporally associated with SARS- CoV-2 infection. He responded well to intravenous methylprednisolone therapy followed by oral prednisolone. While various neurological and ophthalmological manifestations of COVID-19 have been described previously, there are few case reports of Optic neuritis associated with COVID-19. Our case further supports the evidence to suggest that SARS CoV-2 is another such virus that triggers MOG-AD.

\section{Introduction}

Myelin oligodendrocyte glycoprotein (MOG) is a glycoprotein located on the myelin surface in the central nervous system. Optic Neuritis is the most common presentation of MOG Antibody Disease (MOG-AD) seen in $54-61 \%$ of patients and is followed by myelitis. (1). Isolated optic neuritis though rare have higher rate of bilateral presentation in children as compared to adults. Common causes include autoimmune diseases such as systemic lupus erythematosus or MOG-AD, post or para infectious conditions, granulomatous disease, paraneoplastic disorders, and demyelination. (2)

\section{Case Report}

We share our experience with a 11-year-old boy who presented with a rapidly progressive loss of vision. Four days after a brief episode of fever, he complained of redness and painful movement of both eyes which resolved with topical treatment prescribed by his family physician. Two weeks later, he presented to us with a 3-day history of rapidly progressive loss of vision in the right eye. There were no other systemic or neurologic complaints. On physical examination he had bilateral conjunctival congestion, painful eye movement and no perception or projection of light in the right eye. Pupils were bilaterally equal and reactive. Rest of the neurological system including cranial nerves was normal.

Detailed ophthalmological examination revealed a vision of $<\mathrm{N} / 36$ and disc edema with grade 3 Relative Afferent Pupillary Defect in the right eye. Vision in the left eye was $6 / 9$ and rest of the eye examination was within normal limits.

Blood counts revealed hemoglobin $13 \mathrm{~g} / \mathrm{dL}$, white cell count $6.2 \times 10^{3} / \mu \mathrm{L}$ (neutrophils $35 \%$, lymphocytes $44 \%$, monocytes $9 \%$ ), platelets $456 \times 10^{3} / \mu \mathrm{L}$, ESR $13 \mathrm{~mm} /$ hour and CRP was 0 . SARS-CoV-2 nasopharyngeal swab was positive by Cartridge-Based Nucleic Acid Amplification Test (CBNAAT). Serum anti-SARS-CoV-2 IgG antibodies were reactive 20.8 (Normal range $<1.0$ ). Serum myelin Oligodendrocyte Glycoprotein (MOG) antibody was positive and Aquaporin 4 antibody was not detected. CSF showed proteins $10 \mathrm{mg} / \mathrm{dL}$ (normal 15-60 mg/L), glucose $60 \mathrm{mg} / \mathrm{dL}$ (40 - $70 \mathrm{mg} / \mathrm{dL}$ ). CSF white blood cells were elevated at 55 cells $/ \mathrm{mL}$ (normal $<5$ cells $/ \mathrm{mL}$ ). CSF Oligoclonal bands, MOG- IgG and bacterial cultures were negative. CSF- SARS- CoV-2 RNA PCR was not done due to lack of availability. 
MRI findings were consistent with MOG antibody associated disease (3). There was bilateral asymmetrical optic neuritis, right > left, involving the intra orbital and intracanalicular portion on right side and intra orbital portion on the left side. The swelling and altered signal intensity were long segmental and diffuse in the right intra orbital optic nerve and patchy in the left intra orbital optic nerve. There was associated enhancement of the optic nerve sheath in the right orbit. The chiasmatic, retro-chiasmatic portion as well as optic tracts were normal in signal intensity and appearance on both sides. (Figure 1) No restricted diffusion was noted within the optic nerves. Rest of the brain and spine did not show lesions in the white or grey matter. No abnormal brain parenchymal or meningeal post contrast enhancement was seen. We thus concluded a diagnosis of isolated bilateral optic neuritis associated temporally with SARS CoV-2 infection. There was a drastic positive response to 5 days of intravenous methylprednisolone administered at $30 \mathrm{mg} / \mathrm{kg}$ followed by oral prednisolone at $2 \mathrm{mg} / \mathrm{kg} /$ day. Throughout the hospital stay the child remained stable and there was no evolution of any other features. After 10 days of treatment, right eye vision improved to $6 / 9$ and left eye vision was $6 / 6$. Slit lamp and fundus examination was normal. Prednisolone is being slowly tapered over 3 months.

\section{Discussion}

SARS-CoV-2 causes a multipronged multiorgan damage through direct and /or immune mediated injury through release of cytokines. While the respiratory system involvement is the most recognized and common life-threatening manifestation, there is increasing evidence of extra-pulmonary involvement of SARS CoV-2, especially in organs with high angiotensin-converting enzyme 2 (ACE 2) receptor expression including the nervous system and eye (4). Multiple studies have found various ophthalmic manifestations such as hyperaemia, chemosis, epiphora, and increased secretion (5), however conjunctivitis, uveitis, retinitis and optic neuritis have been reported in feline and murine models (6). While, neurological manifestations of hyposmia, hypogeusia, headache, dizziness, altered mental status and GBS have been well established (4), there have been three case reports of SARS CoV-2 associated optic neuritis in a 15 year old boy with anti MOG-IgG positive NMOSD, a 26 years old man with bilateral MOG associated optic neuritis and myelitis and a 29 year old woman with Non MOG associated Optic Neuritis (7-9).Anti MOG associated ON is also frequently associated with viral infections (9-10) and our case adds to the body of evidence that SARS CoV-2 is another such virus that triggers MOG-AD.

\section{Declarations}

\section{Consent and declaration for publication fee:}

A written informed consent was obtained from the family to publish this manuscript.

\section{Funding:}

No specific funding was received from any bodies in the public, commercial or not-for-profit sectors to carry out the work described in this article. 


\section{Conflict of interest:}

Authors declare no conflicts of interest

\section{Contribution of each author:}

Dr Archana Khan, Dr Divya Ramadoss and Dr Raju Khubchandani, were involved in collecting patient's data and in the clinical management of this patient. Dr Archana Khan, Dr Hiren Panwala, Dr Divya Ramadoss and Dr Raju Khubchandani were involved in conducting literature search, drafting the manuscript, and in revision of the scientific content. Dr. Hiren Panwala has conducted and reported on the MRI of brain and spine of this child and drafted parts of the manuscript related to the findings.

\section{Acknowledgement}

Dr Hitendra Mehta diagnosed Optic Neuritis in the child and is currently following up for the same.

S Ophthalmology, F.M.R.F (Vitreoretinal Fellowship Shankar Netralaya, Chennai)

Medical director

Vitreoretinal surgeon

Infiniti Eye Hospital, Spencer Building, Forjett Mumbai 400036, Maharashtra, India

\section{Bibliography}

1. Wynford-Thomas R, Jacob A, Tomassini V. Neurological update: MOG antibody disease. Journal of Neurology. 2018;266(5):1280-1286.

2. Braamskamp M, Dolman K, Tabbers M. Clinical practice. European Journal of Pediatrics. 010;169(10):1179-1185.

3. Denève $M$, Biotti $D$, Patsoura $S$, Ferrier $M$, Meluchova $Z$, Mahieu $L$ et al. MRI features of demyelinating disease associated with anti-MOG antibodies in adults. Journal of Neuroradiology. 2019;46(5):312318.

4. Baig AM, Sanders EC. Potential neuroinvasive pathways of SARS-CoV-2: Deciphering the spectrum of neurological deficit seen in coronavirus disease-2019 (COVID-19). J Med Virol. 2020;2019(May):1845-57.

5. Wu P, Duan F, Luo C, Liu Q, Qu X, Liang L et al. Characteristics of Ocular Findings of Patients With coronavirus Disease 2019 (COVID-19) in Hubei Province, China. JAMA Ophthalmology. 2020;138(5):575.

6. Seah I, Agrawal R. Can the Coronavirus Disease 2019 (COVID-19) Affect the Eyes? A Review of Coronaviruses and Ocular Implications in Humans and Animals. Ocular Immunology and Inflammation. 2020;28(3):391-395. 
7. de Ruijter N, Kramer G, Gons R, Hengstman G. Neuromyelitis optica spectrum disorder after presumed coronavirus (COVID-19) infection: A case report. Multiple Sclerosis and Related Disorders. 2020; 46:102474.

8. Zhou S, Jones-Lopez E, Soneji D, Azevedo C, Patel V. Myelin Oligodendrocyte Glycoprotein AntibodyAssociated Optic Neuritis and Myelitis in COVID-19. Journal of Neuro-Ophthalmology. 2020;40(3):398-402.

9. Palao M, Fernández-Díaz E, Gracia-Gil J, Romero-Sánchez C, Díaz-Maroto I, Segura T. Multiple sclerosis following SARS-CoV-2 infection. Multiple Sclerosis and Related Disorders. 2020; 45:102377.

10. Choi S, Oh D, Chun W, Kim S. The Relationship between Anti-Myelin Oligodendrocyte Glycoprotein Antibody-Associated Disease and the Rubella Virus. Journal of Clinical Neurology. 2018;14(4):598.

11. Koga M., Takahaski T., Kawai M., Fujihara K. A serological analysis of viral and bacterial infections associated with neuromyelitis optica. J. Neurol. Sci. 2011;300(1-2):19-22. Jan 15

\section{Figures}

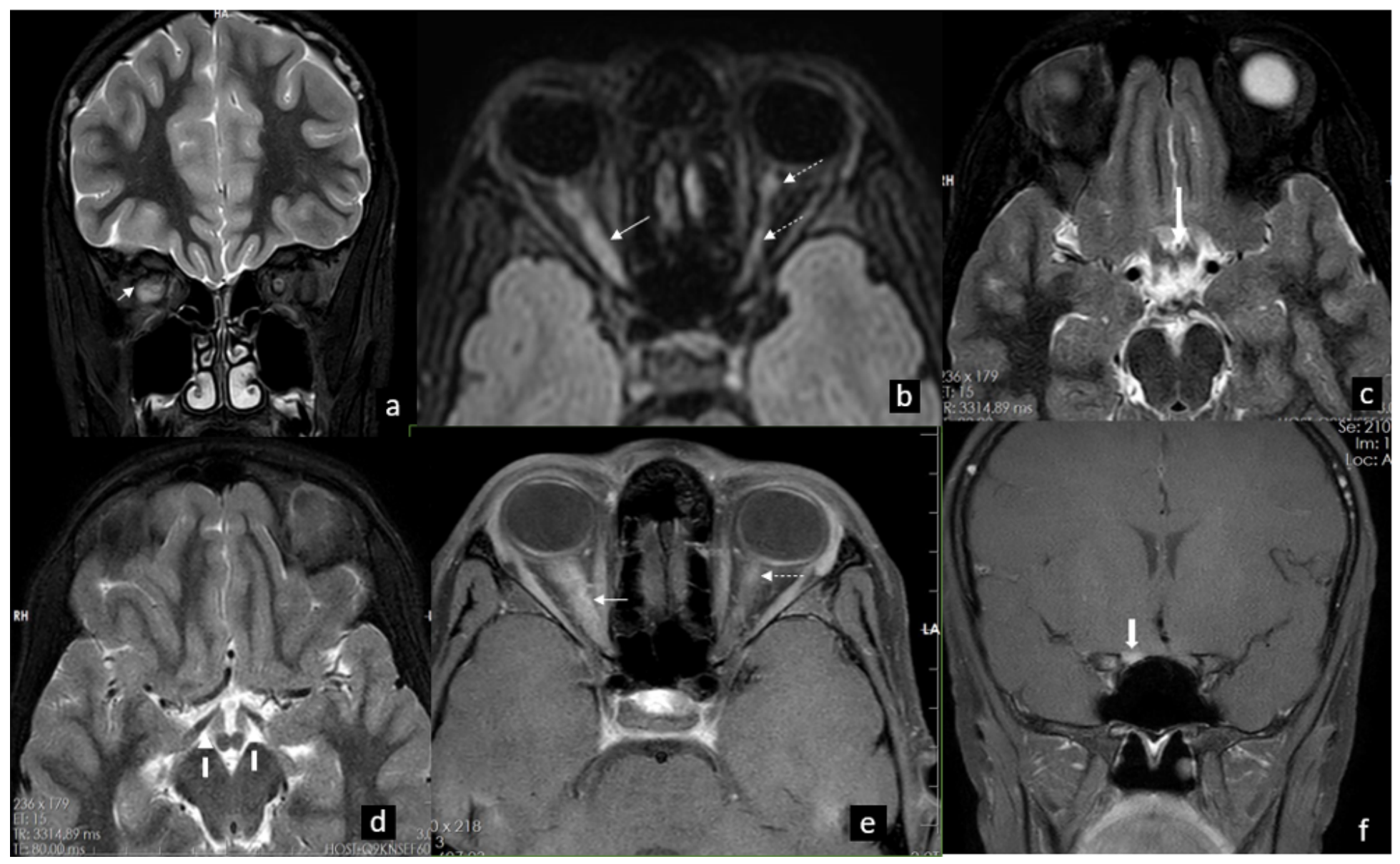

\section{Figure 1}


MRI Brain and Orbits with contrast: (a) STIR coronal images showing swelling and hyperintensity of the right optic nerve (white arrow). (b) FLAIR image showing long segmental swelling and hyperintensity of the intraorbital portion of the right optic nerve (white arrow). Subtle altered signal intensity also noted in the intraorbital portion of the left optic nerve (dashed arrows). (c), (d) T2 W images showing normal appearance of the optic chiasma (thick white arrow) and optic tracts (dashed white arrow). (e, f) T1 W fat saturated post contrast axial and coronal images showing enhancement along the intraorbital (white arrow) and intracanalicular portion (solid white arrow in image (f)) of the right optic nerve. Patchy enhancement (white dashed arrow) also seen in the intraorbital portion of the left optic nerve. No intraconal or extraconal fat stranding or other focal lesions. The extraocular muscles are normal.

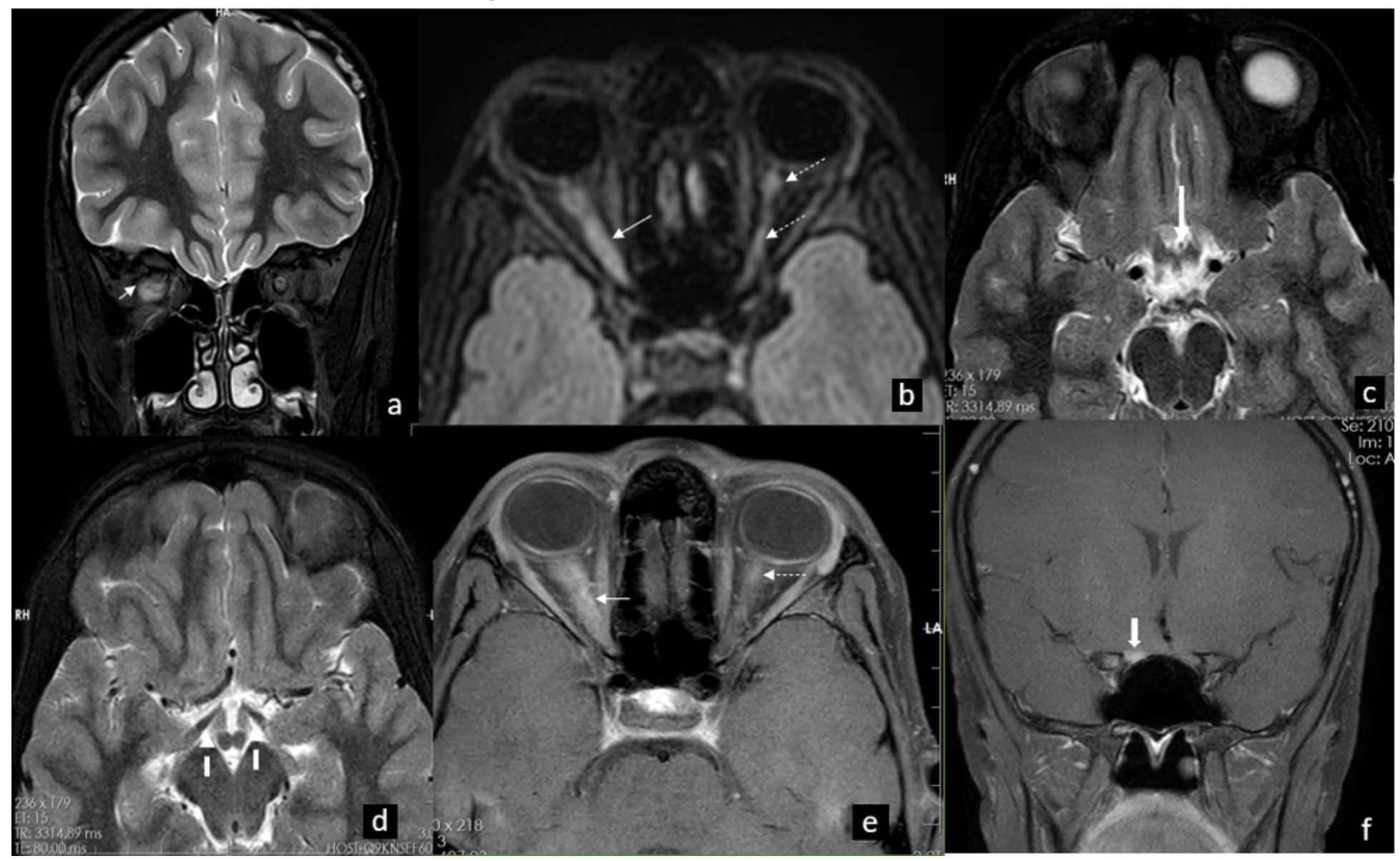

\section{Figure 1}

MRI Brain and Orbits with contrast: (a) STIR coronal images showing swelling and hyperintensity of the right optic nerve (white arrow). (b) FLAIR image showing long segmental swelling and hyperintensity of the intraorbital portion of the right optic nerve (white arrow). Subtle altered signal intensity also noted in the intraorbital portion of the left optic nerve (dashed arrows). (c), (d) T2 W images showing normal appearance of the optic chiasma (thick white arrow) and optic tracts (dashed white arrow). (e, f) T1 W fat saturated post contrast axial and coronal images showing enhancement along the intraorbital (white arrow) and intracanalicular portion (solid white arrow in image (f)) of the right optic nerve. Patchy enhancement (white dashed arrow) also seen in the intraorbital portion of the left optic nerve. No intraconal or extraconal fat stranding or other focal lesions. The extraocular muscles are normal. 


\section{Supplementary Files}

This is a list of supplementary files associated with this preprint. Click to download.

- informationandbibliography.docx

- informationandbibliography.docx 